\title{
ELISIÓN Y SUSTITUCIÓN DE LAS PREPOSICIONES EN ESPAÑOL EN NARRACIONES DE NIÑOS CON TRASTORNO ESPECÍFICO DEL LENGUAJE*
}

\author{
ELISION AND SUBSTITUTION OF PREPOSITIONS IN SPANISH \\ IN NARRATIONS OF CHILDREN WITH SPECIFIC LANGUAGE \\ IMPAIRMENT
}

\section{NINA CRESPO}

Pontificia Universidad Católica de Valparaíso, Valparaíso, Chile. nina.crespo@pucv.cl

\section{WALTER KOZA}

Pontificia Universidad Católica de Valparaíso, Valparaíso, Chile.

walter.koza@pucv.cl

\section{PEDRO ALFARO}

Pontificia Universidad Católica de Valparaíso, Valparaíso, Chile.

pedro.alfaro@pucv.cl

\section{RESUMEN}

Las preposiciones son un problema evidente para los niños diagnosticados con Trastorno Específico del Lenguaje (TEL), quienes en su producción oral tienden a elidirlas o a utilizarlas incorrectamente. Si bien este fenómeno ha sido descrito en la producción lingüística de hablantes de diversas lenguas, en español no ha sido estudiado en profundidad. En este contexto, el presente trabajo busca describir y analizar, de forma cualitativa, los errores en el uso de la preposiciones en niños chilenos diagnosticados con TEL. Para ello se analizaron narraciones orales producidas por 38 niños diagnosticados con TEL que iniciaban el nivel preescolar, entre 5 y 5,11 años de edad. Las narraciones fueron elicitadas a través del recontado y transcritas fonéticamente. Luego se analizaron los contextos de aparición de las preposiciones y se determinó si correspondía a usos adecuados, elisiones o sustituciones de partículas. Los datos indican que existen tres factores gramaticales que parecen incidir en la producción de desviaciones gramaticales en las contribuciones que incorporan preposiciones: (i) la naturaleza funcional de las preposiciones, (ii) la obligatoriedad de los

*El estudio acerca del uso de la preposición en niños con TEL presentado en este artículo se ha desarrollado en el contexto del proyecto de investigación Fondecyt 1160653 “¿El trastorno específico del lenguaje, un déficit en la adquisición y procesamiento de la reglas sintácticas? Pervivencia e influencia en el rendimiento escolar". 
constituyentes y (iii) la incongruencia entre papeles temáticos y funciones sintácticas.

Palabras clave: Trastorno específico del lenguaje, sintagma preposicional, papeles temáticos, funciones sintácticas.

\begin{abstract}
Prepositions represent an evident problem for children diagnosed with Specific Language Impairment (SLI), as they tend to elide prepositions or incorrectly use them in their oral production. This subject has been described in different languages, in Spanish it has not been studied in depth. In this context, the present work seeks to describe and analyze, qualitatively, the errors in the use of prepositions in Chilean children diagnosed with SLI. For this, oral narratives were analyzed produced by 38 children starting preschool between 5 and 5 and 11 month of age, and diagnosed with SLI. Narratives were elided through recounting and were phonetically transcribed. Thereafter, contexts where prepositions appeared were analyzed and it was determined if they matched with adequate use, elisions, or particle substitutions. Data indicate that there are three grammatical factors which seem to influence in the production of grammatical deviations in contributions that incorporate prepositions: (i) the functional nature of prepositions, (ii) the obligatory nature of the components, and (iii) the incongruence between the thematic roles and syntactic functions.
\end{abstract}

Keywords: Specific Language Impairment, thematic role, preposition, syntactic functions.

Recibido: 15.03.2017. Aceptado: 20.04.2018.

\title{
1. INTRODUCCIÓN
}

Zn español, las preposiciones cumplen un rol central en la distribución de las funciones sintácticas dentro de la oración (Fernández Soriano, 1993; Laguna Campos, 2004). No obstante, muchas veces el uso de estas partículas resulta complejo para los hablantes, sean estos nativos, estudiantes de ELE o manifiesten alguna patología asociada al lenguaje. Particularmente, se ha observado que cuando la preposición forma parte de una perífrasis verbal o cuando vincula un sintagma preposicional (SP) y un verbo, sean de calidad núcleo-complemento/argumento o núcleo-adjunto, se suelen ocasionar agramaticalidades. También es posible que influya el carácter funcional y léxico de una determinada preposición, así como la polisemia de muchas preposiciones.

Esta complejidad del sistema preposicional del español deriva en un problema evidente para los niños diagnosticados con Trastorno Específico del Lenguaje (TEL), así en sus producciones orales se observa que, en muchas de sus emisiones, tienden a elidir la preposición (1) o utilizan preposiciones incorrectas (2). 
/don biótes fué su kása/ (sujeto 1)

don bigotes fue [a] su casa

/las maripósas estában xuándo con el párke/ (sujeto 7)

las mariposas estaban jugando con el parque

Esta particularidad del TEL ha sido abordada desde diversas perspectivas (Leonard, 2014).

Por una parte, están los estudios cuantitativos en los que se da cuenta de las diferencias estadísticamente significativas en la cantidad de errores con las preposiciones cometidos por niños con TEL, respecto de la evidenciada por niños con desarrollo típico, como es el caso del estudio de Auza y Morgan (2013) para niños hispanohablantes. Por otra, destacan los estudios descriptivos que intentan dar cuenta de cómo se despliega el sistema preposicional en el TEL, considerando las peculiaridades de cada lengua y las propuestas de gramáticas formales. Dentro de este segundo grupo, dos trabajos se presentan como antecedentes. Por un lado, Roeper, Ramos, Seymour y Abdul Karim (2001) observan el fenómeno en niños con TEL, hablantes de inglés, desde la perspectiva chomskiana, y lo explican como una incapacidad de los sujetos de leer ciertos rasgos categoriales de naturaleza formal. Por otro lado, Mastropavlou y Tsimpli (2011) dan cuenta del fenómeno con niños griegos e indican -a la luz de la teoría minimalista de Chomsky (Chomsky, 1995)- que los errores se observan sobre todo en los elementos que poseen representación fonológica, pero no conceptual. En el marco de este segundo tipo de estudios, la presente investigación buscó dar cuenta en forma sistemática de las elisiones y sustituciones de las preposiciones en el ámbito del sintagma verbal de niños hispanohablantes con TEL. A partir de allí se propuso analizar el fenómeno dentro de la lógica del sistema preposicional del español y contrastar estos hallazgos con las explicaciones formales dadas en el ámbito de otras lenguas.

El artículo se organiza de la siguiente manera. En la sección 2 se presenta el marco teórico que sustenta la investigación. En la sección 3 se describe la metodología. En la sección 4 se discuten los resultados obtenidos. Finalmente, en la sección 5 se presentan las consideraciones finales derivadas de la investigación.

\section{MARCO TEÓRICO}

\subsection{Caracterización del Trastorno Específico del Lenguaje (TEL)}

Dentro de las patologías del lenguaje que pueden afectar la adquisición inicial en algunos niños, una de las más habituales es la que se conoce como Trastorno Específico del Lenguaje (TEL) (Villanueva, De Barbieri, Palomino y Palomino, 2008). 
La prevalencia estimada de este trastorno en la población de habla inglesa oscila entre 2 y 7\% (Tomblin et al., 1997). En Chile, De Barbieri, Maggiolo y Alfaro (1999) señalan que la presencia de TEL alcanza un 4\% entre los niños de 3 y 7 años, con una mayor frecuencia en varones. Esta condición afecta distintos niveles del lenguaje y, por ello, los sujetos con TEL se caracterizan por tener dificultades en la expresión y/o comprensión del lenguaje, aunque no presentan déficits neurológicos, sensoriales, intelectuales o emocionales.

Diversos autores (Bishop y Leonard, 2001; Contreras y Mendoza, 2011) señalan el carácter medular de las dificultades gramaticales en el TEL. En este sentido, se ha observado que los sujetos con dicho trastorno muestran una gran variedad de déficits sintácticos en la producción del lenguaje (Hansson y Nettelbladt, 1995; Thordardottir y Weismer, 2002; Van der Lely y Battel, 2003; Leonard, 2014), entre ellos: en el orden de palabras en la oración (Hansson y Nettelbladt, 1995), en la elaboración de oraciones interrogativas (Van der Lely y Battell, 2003), en el uso de adjuntos y en la representación de elementos gramaticales que dependen de categorías funcionales (Leonard, 2014).

En relación con el español, Contreras y Mendoza (2011) afirman que hay diferentes estudios dirigidos a determinar los problemas gramaticales que experimentan los niños hispanohablantes con TEL; señalan que estos sujetos se caracterizan por un empleo inconsistente de concordancia, uso incorrecto de los nexos, utilización anómala de los morfemas gramaticales y de palabras funcionales, sintaxis desestructurada y de menor nivel de complejidad. Específicamente, en lo que atañe al uso del sistema preposicional (al igual que cualquier elemento funcional) es uno de los aspectos en los que se observa un alto número de desviaciones. Así, por ejemplo, el trabajo de Bedore y Leonard (2005) señala que los preescolares de habla hispana con TEL muestran altos niveles de precisión en las inflexiones de tiempo verbal presente y pasado, que no se dan en niños hablantes de otro idioma. Sin embargo, el uso de artículos definidos, clíticos de objeto directo y preposiciones es significativamente más problemático, observándose en dichos casos sustituciones y omisiones. Los autores concluyen que algunas de las diferencias entre los resultados de español y los de estudios anteriores sobre el inglés o el italiano sugieren la necesidad de procedimientos de evaluación e intervención clínicas que consideren tanto las especificidades del idioma como la tipología de la lengua. Esto se justifica en la medida en que los tipos de error que se dan en una lengua no necesariamente se dan de la misma manera en otra. En relación con ello, puede mencionarse el trabajo de Bortolini et al. (2006), quienes indican que los marcadores lingüísticos de TEL para el italiano no son los mismos que para el inglés, y el trabajo de Leonard (2014), quien -a partir de un estudio que compara varias lenguas- señala que tanto las fortalezas como las debilidades de los niños con TEL están influenciadas por las características de la lengua adquirida. A la vez, un claro ejemplo sería el orden de palabras en inglés 
y en español. Mientras que, para el inglés, el cambio de orden es un indicador de TEL, no lo es para el español, dado que esta lengua permite un orden mucho más libre (Crespo y Sotelo, 2017).

\subsection{Rasgos específicos de la gramática TEL. Hipótesis explicativas}

La elisión y la sustitución de las preposiciones por parte de los niños con TEL, al igual que otros problemas gramaticales, ocurre en diversos idiomas (Van der Lely y Battell, 2003; Puglisi, Befi-Lopes y Takiuchi, 2005; Mastropavlou y Tsimpli, 2011). El intento por dar una explicación lingüística sistemática a este fenómeno ha dado lugar a diferentes teorías, que buscan, por un lado, explicar el carácter específicamente lingüístico del TEL y, por otro, comprender los universales que subyacen a todas las lenguas que resultan afectadas por él. Así, es posible-como se señaló más arriba- que un niño con TEL no manifieste idénticas dificultades al aprender inglés o español (Bedore y Leonard, 2005), sin embargo, estas dificultades podrían estar respondiendo a un factor común que las subyace.

Entre ellos, Roeper et al. (2001) sostienen que los problemas para manejar ciertas categorías gramaticales se relacionan con las dificultades de los niños con TEL para dominar rasgos formales subcategoriales no legibles semánticamente. Así, por ejemplo, estos hablantes no presentan problemas de concordancia entre 'determinante' y 'nombre', puesto que la correlación entre género y número es evidente para ellos. Sin embargo, en el caso del SP, la correlación entre 'verbo' y 'preposición' no es evidente, puesto que no se marca semánticamente, lo que redunda en dificultades para establecer la relación entre ambas unidades.

Por otro lado, Mastropavlou y Tsimpli (2011) entienden las desviaciones gramaticales presentadas por los niños con TEL a partir de la Hipótesis de la Interpretabilidad (HI), formulada inicialmente por Tsimpli y Stavrakaki (1999). Dicha propuesta, basada en la teoría minimalista (Chomsky, 1995) sostiene que los rasgos lingüísticos pueden ser de dos tipos: a) interpretables en la forma lógica, que son más fáciles de aprender, porque, además de su entrada en el lexicón lingüístico, están asociados con rasgos semántico/conceptuales del lexicón mental y son, por definición, no modulares, y b) no interpretables que sirven solamente para funciones morfosintácticas con una representación en la forma fonológica; estos últimos se encuentran en el módulo del lenguaje, lo que dificulta las posibilidades de aprenderlos. En esta perspectiva, los niños con TEL tienen un déficit lingüístico específico porque presentan dificultades para tratar con los rasgos no interpretables, que solo poseen representación fonológica y no conceptual.

En el presente trabajo se propone un análisis de las desviaciones observadas en la construcción de los SP en niños chilenos con TEL, considerando tanto estas 
teorías como la especificidad del español. Por este motivo, en la sección siguiente se describe brevemente las características más sobresalientes de dicha construcción en esta lengua.

\subsection{El sistema preposicional del español}

\subsubsection{El estatus sintáctico/semántico de las preposiciones}

En español, las preposiciones son unidades invariables de número cerrado. Tradicionalmente, han sido definidas a partir de su posición anterior a un 'término', y de su función de subordinarlo a un 'regente'. Desde una perspectiva sintáctica, la preposición y su término son interdependientes, por lo que la ausencia de alguno de ellos causa agramaticalidad. Además, el término es de categoría nominal: sustantivos (he viajado por África), adjetivos en función predicativa (pasarás por cobarde), pronombres personales (para nosotras), verbos en infinitivo (prepararse para comer), adverbios temporales (es de ayer) y espaciales (lo dejó por aqui), oraciones con función nominal junto a la conjunción 'que' (hazlo sin que se den cuenta) y pronombres relativos (...para los cuales) (Fernández López, 1999; Gómez Torrego, 2006). Desde una perspectiva semántica, el 'término' expande el significado de la preposición que la antecede (Fernández Leborans, 2005), por lo que estas relaciones pueden ser de muy variada naturaleza. Esto genera que el inventario cerrado de preposiciones se amplíe mediante la incorporación de locuciones (junto a) y agrupaciones preposicionales (por entre) (Bosque, 1990).

Ahora bien, más allá de esta caracterización, las preposiciones del español constituyen una categoría problemática desde el punto de vista de su caracterización gramatical, debido a la heterogeneidad de los miembros que conforman el paradigma (Minguell, 2009). Desde una perspectiva formal, existe una discusión en torno al carácter léxico, funcional o ambos de estas partículas. Al respecto, Zato (2014) señala que la gramática generativa establece tres posiciones: (i) existen preposiciones que poseen propiedades léxicas y otras sin contenido léxico, sino puramente gramatical; (ii) las preposiciones son categorías funcionales, de acuerdo con la naturaleza de las propiedades que estas codifican (Baker, 2003), y (iii) las preposiciones son una categoría semiléxica, en la medida en que tendrían tanto propiedades léxicas como funcionales.

En la primera de ellas corresponde a la hipótesis clásica del generativismo (Jackendoff, 1977; Chomsky, 1981), que plantea que existen preposiciones léxicas, en la medida en que poseen un significado nocional y que, a la vez, asignan rol temático; y que existen preposiciones funcionales, que solo contienen significado gramatical sin estructura temática. En relación a ello, Fernández Lagunilla y Anula Rebollo (1995) señalan que si bien ambos tipos de preposiciones coinciden en la 
posibilidad de asignar caso, solo las léxicas seleccionan semánticamente a sus complementos (Selección -S) y se configuran como auténticos SP (Minguell, 2009). Así, por ejemplo, la preposición léxica 'sobre' exige un sintagma determinante de especificador y otro que ocupe la posición de complemento. Desde esta perspectiva, las preposiciones funcionales no conforman SP y se requieren únicamente para cumplir con las condiciones de gramaticalidad, tal como grafica la Figura 1.

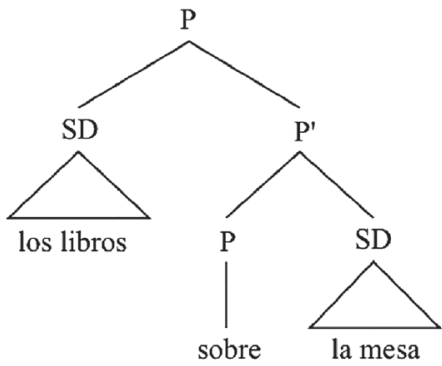

Figura 1. Preposición 'sobre’, adaptado de Halle y Keyser (2002).

En esta línea, Minguell (2009) proporciona los siguientes ejemplos de preposiciones léxicas (2) y funcionales (3):

(2) 2a. Gabriel fue a la facultad. (dirección)

2b. Fabián vivía en Santa Cruz. (localización)

2c. Nicolás viajó con Andrea. (compañía)

(3) 3a. Jorge ama $a$ Valentina.

3b. José insiste en abrigar mucho al bebé.

3c. El informe consta de 12 puntos.

3d. La escritura de la Biblia.

Junto a estas preposiciones funcionales se encuentran además aquellas que se utilizan para conformar perífrasis verbales, las que, como señala Porto Dapena (2002), puede tener valor (4) imperativo (4a), futuro (4b) o pasado (4c).

(4) 4a. Vamos a comer al restaurante.

4b. Mañana voy a comprar pañales.

4c. Ayer fuimos a visitar a María.

Ahora bien, Minguell (2009) señala que en los casos de (2a) y (2b) la preposición encabeza complementos y adjuntos. Al respecto, en el marco de la gramática generativa se han propuesto tres roles para los SP: adjuntos, argumentos y complementos (Neeleman, 1996; 1997; Hamasaki, 2002; Zato, 2014). Los adjuntos 
son constituyentes que no seleccionados semánticamente por el verbo, sino que expresan circunstancias, como por ejemplo:

(5) 5a. Juan y Pedro juegan en el parque.

5b. María lee una revista en el patio de sus suegros.

Por el contrario, los argumentos (locativos) y los complementos son exigidos semánticamente por el verbo. La diferencia principal entre ambos radica en que los primeros tienen un significado espacial (6), mientras que los complementos, significado abstracto (7) (Zato, 2014).

(6) 6a. Lorenzo vive en Rosario.

6b. Luisa puso el pan en la alacena.

(7) 7a. Juan cuenta con que sus amigos le prestarán dinero.

7b. María depende de que sus amigos le presten dinero.

Zato (2014) defiende dicha división a partir de propiedades sintácticas y semánticas, tales como la asignación de caso, el ligamiento, la conmutación, la asignación de rol temático, etcétera.

\subsubsection{La polisemia en el SP}

Como se mencionó, si bien el repertorio de las preposiciones españolas está compuesto por, aproximadamente, 20 unidades, el comportamiento léxico de esta clase de palabras genera que las funciones que ellas desempeñan no se limiten a este número. En efecto, una misma preposición puede tener más de un significado y un significado puede ser expresado por más de una preposición. Otro efecto de esta polisemia es la aparición de otras unidades lexicalizadas y semilexicalizadas que cumplen un rol preposicional: agrupaciones y locuciones preposicionales. Por un lado, las 'agrupaciones' están constituidas por dos o más preposiciones, las que complementan su significado para expresar relaciones sintácticas y semánticas más complejas (caminamos por entre las piedras). En este caso, la primera unidad establece el enlace y la segunda complementa el significado de la primera. Por otro lado, en las locuciones preposicionales se posponen (respecto a) o se anteponen y posponen (a condición de) a sustantivos, adjetivos o adverbios.

Al respecto, diversos trabajos (Omori, 2014; Funes, 2012; Zato, 2014; Ursini y Ticmate, 2016) han señalado el carácter polisémico que poseen las preposiciones con contenido léxico. Así, por ejemplo: 
(8) 8a. Juan está en la sala.

8b. Se comió el alfajor en 5 minutos.

En el caso de 8a, 'en' debe entenderse en un sentido locativo, pues sitúa al sujeto/agente 'Juan' en un lugar. En cambio, en $8 \mathrm{~b}$ la preposición indica la duración en la que se llevó a cabo un proceso.

Dentro de los análisis que abordan la polisemia de las preposiciones, los trabajos de Zato $(2011 ; 2014)$ y Omori $(2014)$ proponen describir este fenómeno a partir de la propuesta de la Teoría del Lexicón Generativo (Pustejovsky, 1995; 2006; 2011). Así, Zato (2011), en un primer momento, establece una estructura de qualia para el sistema preposicional del español, centrando la discusión en torno a los complementos de dichas unidades y añade a dicha estructura el rasgo [+/dinamismo] (Omori, 2014). Posteriormente, Zato (2014) incorpora en su modelo que el significado de los verbos preposicionales se construye composicionalmente a través de un mecanismo léxico de coacción entre regente y preposición.

Por otro lado, Ursini y Ticmate (2016) señalan que la polisemia es un fenómeno gramatical que se debe discutir en arquitecturas gramaticales con componente sintáctico/derivacional independiente y, para ello, argumentan desde la Morfología Distribuida (Halle y Marantz, 1993).

\section{MÉTODO}

Este es un trabajo descriptivo, basado en detección de errores en un corpus de narraciones orales producidas por niños diagnosticados con TEL, a partir de la técnica de recontado (Eisenbeiss, 2010).

\subsection{Sujetos}

La muestra de niños con TEL fue intencionada y estuvo constituida por un total de 38 niños y niñas que asistían a una institución educativa especial para el desarrollo del lenguaje o a un proyecto de integración de las comunas de Valparaíso y Viña del Mar, Chile. Desde allí fueron convocados para su colaboración en esta investigación. Los sujetos pertenecían a los tres estratos socioeconómicos más característicos de Chile, y el indicador de estrato fue el tipo de establecimiento educacional al que asistían: particular, con financiamiento compartido y municipal. En la Tabla I se detallan rasgos de la muestra. 
Tabla I. Distribución de los sujetos por sexo y edad.

\begin{tabular}{|c|c|c|c|c|c|}
\hline \multicolumn{2}{|c|}{ Sexo } & \multicolumn{4}{c|}{ Edad } \\
\hline Niños & Niñas & Mín. & Máx. & Media & Dev. est. \\
\hline 24 & 14 & $5 \mathrm{a} ; 1 \mathrm{~m}$ & $5 \mathrm{a} ; 11 \mathrm{~m}$ & $5 \mathrm{a} ; 4 \mathrm{~m}$ & 0,39 \\
\hline
\end{tabular}

La presencia de TEL en el grupo fue corroborada tras la aplicación del Test Exploratorio de Gramática Española de A. Toronto, subprueba receptiva (STSGR) y subprueba expresiva (STSG-E), en su versión revisada por Pavez (2003), y el TEPROSIF-R (Pavez, Coloma y Maggiolo, 2009). Además, mostraron un desempeño cognitivo normal con la aplicación del Test de Matrices Coloreadas de Raven (2005), siguiendo como criterio la norma chilena (Ivanovich et al., 2000). Junto a ello, se cauteló que tuvieran un desempeño auditivo normal a través de tres procedimientos especializados: otoscopía, audiometría tonal y logoaudiometría. Estos instrumentos fueron aplicados en concordancia con los criterios propuestos en el decreto No 170, basados en el DMS-IV, para detectar el Trastorno Específico de Lenguaje. En la Tabla II se presenta una síntesis de las mediciones realizadas.

Tabla II. Resultados de las evaluaciones aplicadas.

\begin{tabular}{|l|c|c|}
\hline & Media & Dev. est. \\
\hline Pruebas & & \\
\hline STSG-E & 23.4 & 6.64 \\
\hline STSG-R & 24.5 & 7.33 \\
\hline TEPROSIF-R & 30.9 & 14.2 \\
\hline RAVEN & 20.53 & 4.48 \\
\hline Evaluaciones auditivas & Mín. & Máx. \\
\hline Audiometría tonal. Promedio tonal puro oído derecho & 5 & 23.3 \\
\hline Audiometría tonal. Promedio tonal puro oído izquierdo & 3.3 & 21.7 \\
\hline
\end{tabular}

Para salvaguardar los aspectos éticos, los padres, o cuidadores, fueron informados de los objetivos y alcances de esta investigación y firmaron voluntariamente consentimientos informados que autorizaban la participación de sus pupilos. Este proceso fue validado por el Comité de Bioética de la Pontificia Universidad Católica de Valparaíso (receptado con fecha 8 de mayo de 2013). 


\subsection{Recolección de datos y procedimiento de análisis}

Para la construcción del corpus de narraciones orales los sujetos debieron realizar una tarea de recontado, a partir de una historia en formato audiovisual (disponible en https://vimeo.com/91330174).

Dicha historia estaba compuesta por una narración oral (Anexo 1), estructurada en dos episodios, siguiendo la gramática de las historias de Stein y Glenn (1979), adaptada por Peterson y McCabe (1983), Owens (1995) y, en español, por Pavez, Coloma y Maggiolo (2008). Asimismo, el video contenía imágenes estáticas, elaboradas siguiendo la gramática visual de Kress y Van Leeuwen (2008).

Respecto de la aplicación de la tarea, siguiendo a Eissenbeiss (2010) y Reese, Sparks y Suggate (2012), se elaboró un protocolo de presentación de los videos. Así, se presentó el video en forma individual a los participantes, tantas veces como este quisiera y afirmara haber comprendido la historia; luego de ello debieron recontar la historia, utilizando como apoyo un libro álbum que contenía las mismas imágenes del video. Con estos dos procedimientos se buscó minimizar el efecto de la comprensión y la memoria en el recontado. En términos pragmáticos, el procedimiento de presentación del cuento fue realizado por dos evaluadoras especialistas en lenguaje infantil: una que presentaba el video al participante y otra a quien, posteriormente, el participante debió recontar la historia. Con esto se buscó recrear una situación verosímil de narración de historias infantiles.

Cada sesión tuvo una duración aproximada de 15 minutos y fue registrada íntegramente en video. Posteriormente, el recontado oral del sujeto fue transcrito, siguiendo el Alfabeto Fonético Internacional (IPA, 1999).

Para la descripción de los datos se realizó un procedimiento similar al propuesto por el análisis de error aplicado a aprendientes de español como lengua extranjera (Alba Quiñones, 2009). Para llevar a cabo esto, se realizó un conteo de las palabras y de cláusulas que los componían y, posteriormente, se procedió a identificar todos los usos de preposición, diferenciando entre correctos e incorrectos y -dentro de estos últimos- se clasificaron los problemas como elisión o sustitución.

\section{RESULTADOS Y ANÁLISIS}

\subsection{Ocurrencias}

Se identificaron 6.882 palabras y 757 cláusulas. Posteriormente, el análisis se centró en las estructuras elaboradas con preposiciones. En un primer barrido se detectó que de 208 construcciones de preposiciones presentes en el corpus, 13 se relacionaban con sintagmas nominales y 195, con formas verbales. En función 
de esta distribución, se optó por analizar este último grupo, describiendo el comportamiento tanto léxico como funcional de las preposiciones. Asimismo, se reconocieron los usos adecuados y las desviaciones, las que -como se señaló- fueron clasificadas como elisiones o sustituciones.

La Tabla III muestra los resultados generales de los usos preposicionales y de las desviaciones tanto en la conformación de perífrasis verbales como en función de argumentos, complementos y adjuntos (Zato, 2014).

Tabla III. Cantidades y porcentajes de ocurrencia del SVg, tanto en perífrasis como en constituyentes ( $\mathrm{n} / \mathrm{a}=$ no aplica).

\begin{tabular}{|l|c|c|c|c|c|c|c|}
\hline Función & Porcentaje & Papel temático & Tipo & Ocurrencia & Elisión & Sustitución & $\%$ error \\
\hline Perífrasis & $18 \%$ & $\mathrm{n} / \mathrm{a}$ & $\mathrm{n} / \mathrm{a}$ & 35 & 8 & 0 & $22,9 \%$ \\
\hline Complemento & $30 \%$ & Tema & $\mathrm{n} / \mathrm{a}$ & 58 & 17 & 0 & $29,3 \%$ \\
\cline { 3 - 9 } & & Experimentante & $\mathrm{n} / \mathrm{a}$ & 1 & 1 & 0 & $100 \%$ \\
\hline Argumento & $39 \%$ & Lugar & $\mathrm{n} / \mathrm{a}$ & 32 & 3 & 0 & $9,36 \%$ \\
\cline { 3 - 9 } & $13 \%$ & Dirección & $\mathrm{n} / \mathrm{a}$ & 43 & 1 & 2 & $4,65 \%$ \\
\cline { 3 - 9 } & & $\mathrm{n} / \mathrm{a}$ & Lugar & 12 & 3 & 5 & $66,6 \%$ \\
\cline { 3 - 9 } & & $\mathrm{n} / \mathrm{a}$ & Tiempo & 2 & 1 & 0 & $50 \%$ \\
\cline { 3 - 9 } & & $\mathrm{n} / \mathrm{a}$ & Instrumento & 2 & 1 & 0 & $50 \%$ \\
\cline { 3 - 9 } & & $\mathrm{n} / \mathrm{a}$ & Finalidad & 10 & 0 & 0 & $0 \%$ \\
\hline
\end{tabular}

En la Tabla III se evidencia que un 18\% de los usos de preposición aparecen al interior de construcciones verbales perifrásticas. El restante $82 \%$ corresponde a constituyentes de tipo complemento (30\%), argumento (39\%) y adjunto (13\%).

En este sentido, cabe destacar que justamente los dos usos más frecuentes -complementos y argumentos- corresponden a constituyentes requeridos en forma obligatoria por el verbo, mientras que los adjuntos son de uso opcional. Esta tendencia es similar a las que aparecen en el corpus de textos orales producidos por niños con desarrollo típico, que se usó en tareas de comparación y que ha sido descrito en trabajos anteriores (Alfaro-Faccio, Crespo Allende y Alvarado Barra, 2016).

Ahora bien, respecto de las desviaciones se observó que de los 195 usos de la preposición, 160 fueron adecuados, mientras que en 34 casos corresponden a 
elisiones y 5 a sustituciones. Así, el porcentaje de desviaciones en el uso de las preposiciones alcanza el $20 \%$ en la población con TEL estudiada. Dicho porcentaje es bastante mayor que las desviaciones observadas en la población de niños con DT. Esto concuerda con las descripciones de trastorno específico del lenguaje, que lo caracterizan con la presencia de un mayor número de errores gramaticales que las poblaciones típicas (Auza y Morgan, 2013).

En la Tabla III, además, se observan las cantidades totales de elisiones y las sustituciones, así como el porcentaje de error en cada tipo de construcción. En este caso se aprecia que el porcentaje de error varía altamente según cada uno de los tipos de usos preposicionales, desde un $100 \%$ a un $9,36 \%$. En la sección siguiente se analizan cualitativamente estos casos.

\subsection{Análisis}

En el corpus se encontraron 8 casos de elisiones de preposiciones al interior de perífrasis verbales. En todos estos casos las elisiones corresponden a formas verbales con la estructura 'verbo ir + a + verbo infinitivo', en las cuales la preposición cumple la función de vincular las unidades que la componen. Algunas de ellas corresponden a perífrasis con sentido imperativo, como en (9):

(9) /bamóh sabála/ (sujeto 42) vamos [a] salvarla

En estos casos, el sentido imperativo proviene de la forma imperativa "vamos" del verbo "salvar" que, como señala Porto Dapena (1992), corresponde a un verbo español que no tiene una forma imperativa para la primera persona plural.

Otros casos de elisión de la preposición corresponde a perífrasis verbales con sentido futuro, como ejemplifica (10).

(10) /nó bámoh volvér máh a ése lugár/ (sujeto 25) no vamos [a] volver más a ese lugar

Por último, otro caso de este tipo de elisiones corresponde a las perífrasis con un sentido pasado, como ocurre en (11) y en (12)

(11) /xuéron tekatál-la/ (sujeto 7)

fueron [a] rescatarla

(12) /í sús amígah lá fuéron á ír buhkárla/ (sujeto 27)

y sus amigas la fueron a ir [a] buscarla 
Como se observa en todos estos casos, la elisión de esta preposición parece ser sistemática al interior de estas formas verbales, por lo que este fenómeno no parece ser atribuible a cuestiones de orden fónico, como asimilaciones. En estos tres tipos de perífrasis parecen no ser incorporadas las preposiciones que no cumplen una función asociada a su valor léxico.

En cuanto a las elisiones que ocurren a nivel de constituyentes, cabe hacer una comparación entre complementos y argumentos, unidades exigidas por el verbo, es decir, de carácter obligatorio, y los adjuntos, unidades opcionales. Un hecho evidente que muestra la Tabla III consiste en la menor proporción de errores en argumentos y complementos frente a los adjuntos. De esta manera, el complemento tema presenta un 29,3\% de error, los 'argumentos de dirección' y 'lugar' un 9,36 y un $4,65 \%$, respectivamente, mientras que los 'adjuntos de lugar', 'tiempo' e 'instrumento' presentan un $50 \%$ de error cada uno. Dos casos escapan a esta tendencia: el 'argumento con papel temático de experimentante', con un 100\% de error y el 'adjunto con valor de finalidad' con $0 \%$ de error. A continuación realizaremos un análisis detallado de lo que ocurre con esta diferencia y, posteriormente, se observarán los dos casos que se apartan de la tendencia intentando explicar el porqué.

De todas las categorías analizadas, el mayor número de elisiones correspondió a la preposición 'a' en los complementos acusativos con papel temático de tema. Concretamente, este fenómeno afectó a 17 de 58 complementos de este tipo. En estos casos la preposición se utiliza para individualizar referentes correspondientes a personas o animales, como muestran (13), (14) y (15).

(13) Ií etónses lá atapó flópi/ (sujeto 33) y e[n]tonces la atapó Flopi

(14) /fue atlapá mariposa/ (sujeto 4) fue [a] atrapar mariposa[s]

(15) /miméro atlapó la maipósa el kabaéro/ (sujeto 15) primero atrapó la maiposa el cabaero

Como se observa en (13), el uso de las preposiciones es completamente obligatorio, dado que la presencia del pronombre acusativo 'la' exige que el complemento acusativo duplicado sea particularizado a través de la preposición. En el caso de (14), se observa la elisión tanto de la preposición como del determinante del sintagma, es decir, las partículas funcionales involucradas. Finalmente, las elisiones en (15) corresponden a preposiciones cuyo uso podría ser considerado optativo en español, ya que no es del todo claro, en los ejemplos hallados, si corresponde a referentes individualizados. No obstante, en este caso, dada las características del texto que sirvió de modelo a la producción del recontado, el referente 'mariposa', mencionado al inicio del relato, exige la preposición 'a' que lo individualice. 
Por otro lado, en cuanto a los 'argumentos de lugar' y 'dirección', cabe señalar que las desviaciones corresponden tanto a elisiones como a sustituciones de preposiciones. En este caso, las preposiciones afectadas fueron 'a' y 'en', y se encontraron 4 casos de elisión y 2 de sustitución, los que se ejemplifican en (16) y (17).

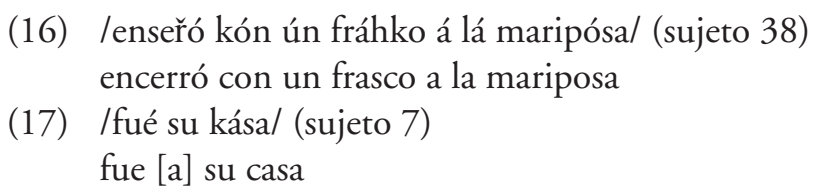

Finalmente, en cuanto a los 'adjuntos de lugar', 'tiempo' e 'instrumento' cabe señalar que las desviaciones correspondían también a elisiones y a sustituciones. En este caso, las preposiciones afectadas también fueron 'a' y 'en', como ejemplifican (18), (19), (20) y (21).

(18) /póke ió á káKe éra úna komét/ (sujeto 22) porque vio[en] la calle, era una comet[a]

(19) /guéla aKí él siélo/ (sujeto 21) vuela ahí [en] el cielo

(20) /ún día flópi i u amía ehtabán xugándo por el párke/ (sujeto 34) un dia Flopi y $[s] \mathrm{u}[\mathrm{s}]$ amigas estaban jugando por el parque

(21) /í tódah suh amígh tában xugándo kón él páhke/ (sujeto 7) y todas sus amigas estaban jugando con [en] el parque

(22) /atrapó la red/ (sujeto 3) atrapo [con] la red

En estos casos, se observa que las preposiciones con contenido léxico 'con' y 'en' pueden tanto ser elididas como cambiadas; en contraposición, las preposiciones funcionales solo son elididas, como ocurre con 'a'. Lo anterior parece deberse a la obligatoriedad del constituyente en aquellos sintagmas preposicionales que indican lugar. Así, mientras son obligatorios -como en el caso de los argumentos locativos- las desviaciones se corresponden con la elisión, mientras que en calidad de adjunto se observan tanto elisiones como sustituciones.

Junto a ello se observó que en los adjuntos ocurren dos casos de elisión o sustituciones de locuciones: la preposicional (23) y la adverbial (24)

(23) / ké lo estába biéndo de un árbol/ (sujeto 35) que lo esta viendo de[sde] un árbol

(24) latí ké lá/élá/ál ekotáte kón lá maepóta nébo/ (sujeto 10) así [es] que fue a e[n]co[n]t[r]a[rs]e con la mariposa [de] nuevo 
Ahora bien, como se mencionó, hubo dos casos que escapan a esta tendencia: un argumento que se utiliza escasamente y con alto índice de error, y un adjunto que se utiliza en diversas ocasiones y sin errores. Concretamente, el primero de ellos tiene una sola ocurrencia y en esta se elide la preposición, y corresponde a una construcción inacusativa de predicado psicológico, propia del español (Marín, 2016), como muestra

(25) /él nó lé gustába ke las marepósas boláran/ (sujeto 44)

[a] él no le gustaba que las mariposas volaran

En este caso, el sujeto 'que las mariposas volaran' representa a la causa de la reacción psicológica y el dativo 'le' corresponde al experimentante de esa reacción. Este tipo de oraciones presentan una incongruencia entre el papel temático y la función sintáctica, lo cual podría ser la causa del error.

Cabe destacar que en el texto modelo para el recontado aparecía la oración "A Don Bigotes no le gustaba que las mariposas volaran en su campo" y que 37 de los 38 niños evaluados, al intentar reproducir el contenido proposicional, utilizaron otro tipo de oraciones o evitaron reproducir el contenido psicológico de la narración.

El grupo de sujetos que utilizó una estructura alternativa corresponde a 6 niños, quienes recurren a lo que Jensen, Sundahl y Chondrogianni (2014) denominan estrategia de evitación, que consiste en eludir los tipos oracionales que tienden a ser complejos para la población con TEL. En efecto, en este caso gran parte de los niños optó por construir estructuras acusativas, semánticamente equivalentes, mediante, por ejemplo, verbos que expresan deseo, como muestra (26).

(26) /no kería las madipósas estubiédan en su kámpo/ (sujeto 13) no quería [que] las mariposas estuvieran en su campo

Finalmente, el grupo de niños que omitió la información psicológica del personaje también es llamativo, dado que esa cláusula en particular portaba la información que permite comprender las acciones del personaje, lo que, a su vez, desencadena el primer episodio de la narración.

Ahora bien, dentro del marco que se ha delineado para las preposiciones dentro de los adjuntos, la partícula 'para' constituye una excepción. En el texto fuente aparecía la expresión "Don Bigotes buscó una red para atrapar a la mariposa"; en este caso 10 de los sujetos evaluados utilizaron la preposición y no la elidieron ni cambiaron por otra. Una posible explicación para este fenómeno puede estar dada por el contenido léxico de la partícula, más que a la función que cumple en esta oración, cuyo valor de finalidad parece ser accesible a los sujetos desde tempranas edades (Peronard, 1987), lo que generaría que no se observen desviaciones en 
sintagmas preposicionales que involucran a esta partícula, como ejemplifica (27).

(27) /i luégo él sejóh fué a bokáh úna řé pára atakáhla/ (sujeto 9) y luego el señor fue a buscar una re[d] para at[r]acpa[r]la

A la vez, en todos los casos aparece con contenido léxico, en contraposición a 'a', que, como se ha mencionado, puede presentar un valor tanto léxico como funcional $y$, a la vez, presenta una polisemia mucho más compleja.

\section{CONSIDERACIONES FINALES}

A través de los ejemplos que hemos presentado, se ha podido observar que los fenómenos de elisión y sustitución que afectan a las preposiciones de los niños hispanohablantes con TEL parecieran ocurrir bajo tres condicionantes: (i) la naturaleza léxica/funcional de las preposiciones; (ii) la obligatoriedad de los constituyentes, y (iii) la incongruencia entre papeles temáticos y funciones sintácticas.

Respecto de (i), se observó que los sujetos eliden preposiciones cuando el valor de estas partículas es puramente funcional, lo que no ocurre cuando, al formar parte de otro contexto, poseen un valor semántico. Esto implica evidencia empírica en favor de dos propuestas. La primera de ellas es el planteo de Mastropavlou y Tsimpli (2011), quienes señalan, a partir de estudios de niños con TEL grecoparlantes, que las categorías no interpretables en la forma lógica son invisibles para estos sujetos. Esto podría explicar las elisiones tanto al interior de las perífrasis verbales como en la marcación del acusativo con la partícula 'a' en español. La segunda indicaría que la dicotomía entre preposiciones léxicas y funcionales no es absoluta, ya que los valores de estas partículas podrían depender de los contextos sintácticos en los que aparecen.

Ahora bien, es necesario aclarar que las elisiones de las preposiciones funcionales no se produjeron en un cien por ciento de los casos, y que incluso un mismo sujeto a veces omitió una de estas partículas y otras no, tal como se observa en (28a) y en (28b).

$$
\begin{aligned}
& \text { (28a) /i e señó bio babiosa/ (sujeto 5) } \\
& \text { y el señor vio [a la] mariposa } \\
& \text { (28b) /la atakó a ella] (sujeto 5) } \\
& \text { y la atacó a ella }
\end{aligned}
$$

Estos hallazgos están en sintonía con la descripción de Leonard (2014), para quien el TEL no constituye una patología uniforme, sino que manifiesta un amplio rango de variabilidad tanto intrasujeto como intersujeto, por lo que no es de 
extrañar que, dependiendo del contexto comunicativo y la exigencia de la tarea, los errores no sean sistemáticos, cuestión que justamente dificulta el estudio de esta patología.

Otra explicación para los errores gramaticales en el TEL, como señalamos, es la proporcionada por Roeper et al. (2001), quienes argumentan que estos se relacionan con el manejo de rasgos formales subcategoriales, vinculados con el dominio de la concordancia morfosintáctica. Sin embargo, esta explicación no parece ser adecuada para el español, dado que en esta lengua la concordancia se establece a través de marcas morfológicas explícitas de género, de número, de persona, etc., en la cuales los sujetos TEL fallan en menor medida. A pesar de ello, efectivamente algunas preposiciones, como 'a' y 'de' cumplen la función de marcar papeles temáticos. Desde esta perspectiva, el fenómeno de la partícula 'a' podría ser, entonces, interpretado desde ambas propuestas, dado que no tiene contenido semántico, y de acuerdo con ello, esta desviación correspondería a un fenómeno morfológico de naturaleza post-sintáctica (Halle y Marantz, 1993).

En cuanto a (ii), es decir, a la obligatoriedad de algunos constituyentes, se observó que las preposiciones con contenido léxico podían ser tanto elididas como cambiadas por otras de contenido similar. En cambio, las preposiciones funcionales no sufrieron sustituciones, solo elisiones. Es interesante esta diferencia en el comportamiento sintáctico de estas partículas, pues permite comprender cómo se distinguen ambas en cuanto a su uso y descripción.

También es pertinente la distinción señalada por Zato (2014), Neeleman (1996; 1997) y Hamasaki (2002), entre los complementos y los argumentos locativos. Al respecto, cabe destacar la preposición 'a', en la que se observan tanto valores funcionales -marca de acusativo, formante de perífrasis- como léxicos -dirección, locación- en el que incluso presenta un alto grado de polisemia, siendo una de las preposiciones con mayores posibilidades de significado. Esta complejidad intrínseca de esta partícula podría explicar la dificultad que representa para los hispanohablantes con TEL. Una propuesta un tanto diferente es el estudio realizado por Littlefield (2006) para el inglés en el proceso de adquisición de niños con desarrollo típico, quien propone una descomposición de los valores [+/- léxico], [+/- funcional] e indica casos en los que ambos polos pueden combinarse. Más allá de que escapa a los objetivos del presente artículo, se hace pertinente un análisis detallado de ambas propuestas, lo que será considerado en trabajos futuros.

En cuanto a (iii), es decir, a la discordancia entre funciones sintácticas y papeles temáticos, parecería adecuado afirmar que los niños con TEL no realizan una lectura cruzada de papeles y funciones (Marín, 2016), sino que asumen una uniformidad en la asignación de estos y marcan al experimentante dativo como un experimentante sujeto, como suele ocurrir en la formas no marcadas del español. Esta asignación que parece ser la más usual en español podría explicar también por qué al parafrasear la oración, la reemplazan por una con verbo acusativo. 
Ahora bien, este fenómeno podría ser explicado desde dos perspectivas. Por un lado, este tipo de predicados psicológicos son estructuras menos frecuentes en la lengua, por lo que los hablantes las aprenden más tarde, tal como ocurre con la voz pasiva. En este caso los niños con TEL evitan esta estructura o fallan al construirla porque no la conocen suficientemente. Por otro lado, podría afirmarse que la coincidencia entre ciertas funciones sintácticas con determinados papeles temáticos -por ejemplo 'sujeto' y 'agente'- haría más sencilla su utilización, ya que el papel temático estaría dando más información y reafirmando la función sintáctica, que es de naturaleza más abstracta. Esta segunda propuesta se aproxima a la propuesta de Tsimpli y Stavrakaki (1999). Creemos que para poder validar alguna de estas perspectivas, es necesario realizar trabajos futuros tanto en el área de los estudios de corpus como en los trabajos experimentales.

\section{REFERENCIAS}

Alba Quiñones, Virginia. (2009). El análisis de errores en el campo del Español como Lengua Extranjera: Algunas cuestiones metodológicas. Revista Nebrija de Lingüistica Aplicada, 5 (3), 1-16.

Alfaro-Faccio, Pedro, Crespo Allende, Nina y Alvarado Barra, Carola. (2016). Complejidad sintáctica en narraciones de niños con desarrollo típico, trastorno específico del lenguaje y discapacidad intelectual. Sintagma, 28, 27-41.

Auza, Alejandra y Morgan, Gareth. (2013). Uso de preposiciones en el recuento de una historia. Comparación de niños hispanohablantes con y sin trastorno del lenguaje. Infancia y Aprendizaje, 36 (1), 35-49.

Baker, Mark. (2003). Lexical Categories. Verbs, Nouns and Adjectives. Cambridge, United Kingdom: Cambridge University Press.

Bedore, Lisa y Leonard, Laurence. (2005). Verb inflections and noun phrase morphology in the spontaneous speech of Spanish-speaking children with specific language impairment. Applied Psycholinguistics, 26 (2), 195-225.

Bishop, Dorothy y Leonard, Laurence. (2001). Speech and language impairments in children: causes, characteristics, intervention and outcome. Oxford, United Kingdom: Psychology Press.

Bortolini, Umberta, Arfé, Bárbara, Caselli, Cristina M., Degasperi, Luisa, Deevy, Patricia y Leonard, Laurence. B. (2006). Clinical markers for specific language impairment in Italian: the contribution of clitics and non word repetition. International Journal of Language \& Communication Disorders, 41, 695-712.

Bosque, Ignacio. (1990). Las categorías gramaticales. Madrid, España: Síntesis.

Chomsky, Noam. (1981). Lectures on Government and Biding. Dordrecht, Netherlands: Foris. 
Chomsky, Noam. (1995). The minimalist program. Cambridge, United States: MIT Press.

Contreras, María del Carmen y Mendoza, Elvira. (2011). El rastreo sintáctico y la planificación de sintagmas: diferencias entre el Trastorno Específico del Lenguaje y la deprivación sociocultural. Revista de Logopedia, Foniatría y Audiología, 31 (1), 47-56.

Crespo, Nina y Sotelo, Eva. (2017). Orden sintáctico y TEL: cómo se observa el fenómeno en español. XXII Congreso Internacional de la Sociedad Chilena de Lingüistica, Talca, Chile.

De Barbieri Zulema, Maggiolo, Mariangela, Alfaro Selma. (1999). Trastornos del lenguaje en una población de niños asistentes a control sano. Revista Chilena de Pediatría, 70, 36-40.

Eisenbeiss, Sonja. (2010). Production methods in language acquisition research. En E. Blom y S. Unsworth (Eds.). Experimental methods in language acquisition research, 11-34. Amsterdam: John Benjamins.

Fernández Leborans, María Jesús. (2005). Los Sintagmas del Español. II El sintagma verbal y otros. Madrid, España: Arco Libros, S.L.

Fernández López, María del Carmen. (1999). Las Preposiciones del Español. Salamanca, España: Ediciones Colegio de España.

Fernández Soriano, Olga. (1993). Sobre el orden de las palabras en español. DICENDA. Cuadernos de Filología Hispánica, 11, 113-152.

Fernández Lagunilla, Marina y Anula Rebollo, Alberto. (1995). Sintaxis y cognición: introducción al conocimiento, el procesamiento y los déficits sintácticos. Madrid, España: Editorial Síntesis.

Funes, María Soledad. (2012). El significado de la preposición 'de': un enfoque cognitivo-prototípico. Boletín de Filología, XLVII (1), 111-135.

Gómez Torrego, Leonardo. (2006). Hablar y Escribir correctamente. Gramática normativa del Español (Vol. II). Madrid, España: Arco/Libros, S.L.

Halle, Ken y Keyser, Samuel Jay. (2002). Prolegomenon to a Theory of Argument Structure. Cambridge, United States: MIT Press.

Halle, Morris y Marantz, Alec. (1993). Distributed Morphology and the Pieces of Inflection. En K. Halle y S. J. Keyser (Eds.) The View from Building 20: Essays in Honor of Sylvain Bromberger, 111-176. Cambridge, United States: MIT Press.

Hamasaki, Michiyo. (2002). A Note on PP-Complements and F Role Assignment. 愛知教育大学研究報告. 人文・社会科学, 51, 113-120.

Hansson, Kristina y Nettelbladt, Ursula. (1995). Grammatical characteristics of Swedish children with SLI. Journal of Speech and Hearing Research, 38, 589598.

IPA (International Phonetic Association). (1999). Handbook of the International Phonetic Association: A guide to the use of the International Phonetic Alphabet. 
Cambridge, United Kingdom: University Press.

Ivanovich, Rodolfo, Forno, Hernán, Durán, María Cristina, Hazbún, Julia, Castro, Carmen e Ivanovich, Daniza. (2000). Estudio de la capacidad intelectual (Test de Matrices Progresivas de Raven) en escolares chilenos de 5 a 18 años. I. Antecedentes generales, normas y recomendaciones. Revista de psicología general y aplicada: Revista de la Federación Española de Asociaciones de Psicología, 53, 5-30.

Jackendoff, Ray. (1977). X-bar syntax: A study of Phrase Structure. Cambridge, United States: MIT Press.

Jensen De López, Kristine, Sundahl Olsen, Lone y Chondrogianni, Vasilike. (2014). Annoying Danish relatives: comprehension and production of relative clauses by Danish children with and without SLI. Journal of Child Language, 41, 51-83.

Kress, Gunther y Van Leeuwen, Teun. (2008). Visual grammar. London, United Kingdom: Equinox.

Laguna Campos, José. (2004). Gramática de las preposiciones. Actas del XIV Congreso Internacional de ASELE. Zaragoza, España.

Leonard, Laurence. (2014). Children with specific language impairment and their contribution to the study of language development. Journal of child language, 41 (1), 38-47.

Littlefield, Heather. (2006). Syntax and Acquisition in the Prepositional Domain: Evidence from English for Fine-Grained Syntactic Categories. Tesis de Doctorado en Applied Linguistics. Boston, Estados Unidos: Boston University Press. Marín, Rafael. (2016). Los predicados psicológicos. Madrid, España: Visor Libros.

Mastropavlou, María y Tsimpli, Ianthi Maria. (2011). Complementizers and subordination in typical language acquisition and SLI. Lingua, 121, 442-462.

Minguell, Antonia. (2009). La preposición: categoría léxica y funcional. Aportes para la enseñanza del español. SIGNOS ELE: Revista de español como lengua extranjera, 3, 1-20.

Neeleman, Ad. (1996). PP-complements in Dutch. Proceedings of West Coast Conference on Formal Linguistics 15, Irvine, Estados Unidos.

Neeleman, Ad. (1997). PP-complements. Natural Language and Linguistic Theory, 15, 89-137.

Omori, Hiroko. (2014). ¿Cómo explicar la polisemia de las preposiciones en el lexicón generativo? Una propuesta para la enseñanza del español desde la teoría lingüística. En N. Contreras. (Ed.). La enseñanza del español como LE/L2 en el siglo XXI, 543-550. Congreso Internacional ASELE.

Owens, Robert. (1995). Language disorders: A functional approach to assessment and intervention. Boston, Unites States: Allyn and Bacon.

Pavez, María Mercedes. (2003). Test exploratorio de gramática española de A. Toronto. Santiago, Chile: Ediciones UC. 
Pavez, María Mercedes, Coloma, Carmen Julia y Maggiolo, Mariangela. (2008). El desarrollo narrativo en niños. Una propuesta práctica para la evaluación y la intervención en niños con trastornos de lenguaje. Barcelona, España: Lexus.

Pavez, María Mercedes, Maggiolo, Mariangela y Coloma, Carmen Julia. (2009). Test para evaluar procesos de simplificación fonológica. TEPROSIF-R. (3ra. Edic.). Santiago, Chile: Ediciones UC.

Peronard, Marianne. (1987). La expresión temporal en las primeras etapas de la adquisición de la lengua materna. En M. Peronard, El lenguaje, un enigma. 7598. Valparaíso, Chile: Ediciones Quijote.

Peterson, Carole y McCabe, Allyssa. (1983). Developmental psycholinguistics: Three ways of looking at a child's narrative. New York, United States: Plenum Press.

Porto Dapena, José Álvaro. (1992). Del indicativo al subjuntivo: valores y usos de los modos del verbo. Madrid, España: Arco/Libros, S.L.

Porto Dapena, José Alvaro. (2002). Complementos argumentales del verbo: directo, indirecto, suplemento y agente. Madrid, España: Arco/Libros, S.L.

Puglisi, María Leite, Befi-Lopes, Debora María y Takiuchi, Noemi. (2005). Utilização e compreensão de preposições por crianças com distúrbio específico de linguagem. Pró-Fono Revista de Atualização Científica, 17 (3), 331-344.

Pustejovsky, James. (1995). The generative lexicon. London, United Kingdom: The MIT Press.

Pustejovsky, James. (2006). Type theory and lexical decomposition. Journal of Cognitive Science, 7 (1), 39-76.

Pustejovsky, James. (2011). Coercion in a general theory of argument selection. Linguistics, 49 (6), 1401-1431.

Raven, John C. (2005). Test de matrices progresivas. Escala coloreada. Cuaderno de Matrices/Series $A, A B$ y $B$. Buenos Aires, Argentina: Paidós.

Reese, Elaine, Sparks, Alison y Suggate, Sebastian. (2012). Assessing children's narratives. En E. Hoff, Research methods in child language. A practical guide. New York, United States: Wiley-Blackwell. 133-148.

Roeper, Thomas, Ramos, Eliane, Seymour, Harry y Abdul-Karim, Lanya. (2001). Language disorders as a window on Universal Grammar: An abstract theory of agreement for IP, DP, and V-PP. Brain and Language, 77 (3), 378-397.

Stein, Nancy y Glenn, Christine. (1979). An analysis of story comprehension in elementary school children, en R. O. Freedle (ed.), Advances in discourse processes: New directions in discourse processing, 53-120. Norwood, United States: Ablex.

Thordardottir, Elain y Weismer, Susan Ellis. (2002). Verb argument structure weakness in specific language impairment in relation to age and utterance length. Clinical Linguistic. Phonology, 16, 233-250.

Tomblin, Bruce, Records, Nancy, Buckwalter, Paula, Zhang, Xuyang, Smith, Elaine y O'Brien, Marlea. (1997). Prevalence of specific language impairment in 
kindergarten children. Journal of speech language and hearing research, 40 (6), 1245-1260.

Tsimpli, Ianthi y Stavrakaki, Stavroula. (1999). The effects of a morpho-syntactic deficit in the determiner system: The case of a Greek SLI child. Lingua, 108, 31-85.

Ursini, Francesco y Ticmate, Adriano. (2016). On the polysemy of spanish spatial Ps. Borealis. An International Journal of Spanish Linguistics, 5 (2), 253-312.

Van der Lely, Heather y Battell, Jackie. (2003). Wh-movement in children whith grammatical SLI: A test of the RDDR hypothesis. Language, 79 (1), 153-179. Villanueva, Pía, De Barbieri, Zulema, Palomino, Hernán y Palomino, Hernán. (2008). Alta prevalencia de trastorno específico de lenguaje en isla Robinson Crusoe y probable efecto fundador. Revista Médica Chilena, 136, 186-192.

Zato, Zoltan. (2011). Alternancia preposicional en los complementos preposicionales regidos en español: Aproximación subléxica. Círculo de Lingüistica Aplicada a la Comunicación, 48, 41-76.

Zato, Zoltan. (2014). Qualia structure in Spanish prepositional verbs: when the verb resorts to a preposition. Borealis. An International Journal of Spanish Linguistics, 3 (1), 49-81. 
ANEXO 1. Estructura y texto de cuento fuente: La mariposa Flopi.

\begin{tabular}{|c|c|}
\hline $\begin{array}{l}\text { Establecimiento } \\
\text { de la escena }\end{array}$ & $\begin{array}{l}\text { Había una vez una Mariposa llamada Flopi. A Flopi le } \\
\text { gustaba salir a bailar por el viento y a pasear con sus ami- } \\
\text { gas. Todos los días daban una vuelta por el parque y se } \\
\text { divertían mucho cantando y riendo. }\end{array}$ \\
\hline Evento inicial & $\begin{array}{l}\text { Un día, Flopi y sus amigas fueron muy contentas a volar } \\
\text { por el campo lleno de flores y frutas. El campo era de un } \\
\text { señor muy enojón, llamado Don Bigotes. } \\
\text { Estaban volando sobre las margaritas y, de repente, sintie- } \\
\text { ron que alguien las miraba detrás del árbol. }\end{array}$ \\
\hline $\begin{array}{l}\text { Respuesta interna } \\
\text { al estado }\end{array}$ & $\begin{array}{l}\text { ¡Era Don Bigotes aburrido de que las mariposas jugaran } \\
\text { en su campo! }\end{array}$ \\
\hline Plan & Voy a cazarlas, pensó. \\
\hline Intento & $\begin{array}{l}\text { Fue a su casa y buscó su red para atrapar mariposas. Una } \\
\text { vez de vuelta en el campo comenzó a perseguir a las ma- } \\
\text { riposas. Entonces Flopi y sus amigas empezaron a volar } \\
\text { rápido y más rápido. }\end{array}$ \\
\hline $\begin{array}{l}\text { Consecuencia } \\
\text { directa }\end{array}$ & $\begin{array}{l}\text { Pero Flopi no pudo escapar y fue atrapada por el Señor } \\
\text { Bigotes. }\end{array}$ \\
\hline Evento inicial & Él se la llevó a su casa y la encerró en un frasco. \\
\hline $\begin{array}{l}\text { Respuesta interna } \\
\text { al estado }\end{array}$ & $\begin{array}{l}\text { Flopi estaba muy triste y asustada porque no podía volar } \\
\text { ni moverse. }\end{array}$ \\
\hline plan & $\begin{array}{l}\text { Sus amigas preocupadas se pusieron a conversar. ¿Qué } \\
\text { haremos?, se preguntaban. ¡Debemos salvarla sí o sí! Es- } \\
\text { peremos que se duerma don Bigotes y vamos a rescatarla. }\end{array}$ \\
\hline Intento & $\begin{array}{l}\text { Don Bigotes se fue a dormir y las mariposas amigas entra- } \\
\text { ron por la ventana a la casa. Todas juntas giraron la tapa } \\
\text { del frasco y liberaron a Flopi. }\end{array}$ \\
\hline $\begin{array}{l}\text { Consecuencia } \\
\text { directa }\end{array}$ & $\begin{array}{l}\text { Flopi y sus amigas huyeron rápidamente de la casa de } \\
\text { Don Bigotes y volaron para alejarse del campo. }\end{array}$ \\
\hline $\begin{array}{l}\text { Reacción y } \\
\text { resolución }\end{array}$ & $\begin{array}{l}\text { Las mariposas estaban muy contentas y prometieron } \\
\text { nunca más volver a ese lugar. }\end{array}$ \\
\hline
\end{tabular}

